\title{
Double-blind, randomized pilot clinical trial targeting alpha oscillations with transcranial alternating current stimulation (tACS) for the treatment of major depressive disorder (MDD)
}

Morgan L. Alexander ${ }^{1,2}$, Sankaraleengam Alagapan ${ }^{1,2}$, Courtney E. Lugo ${ }^{1}$, Juliann M. Mellin ${ }^{1,2}$, Caroline Lustenberger ${ }^{1,3}$, David R. Rubinow ${ }^{1}$ and Flavio Fröhlich (1) $1,2,4,5,6,7$

\begin{abstract}
Major depressive disorder (MDD) is one of the most common psychiatric disorders, but pharmacological treatments are ineffective in a substantial fraction of patients and are accompanied by unwanted side effects. Here we evaluated the feasibility and efficacy of transcranial alternating current stimulation (tACS) at $10 \mathrm{~Hz}$, which we hypothesized would improve clinical symptoms by renormalizing alpha oscillations in the left dorsolateral prefrontal cortex (dIPFC). To this end, 32 participants with MDD were randomized to 1 of 3 arms and received daily 40 min sessions of either $10 \mathrm{~Hz}$ tACS, $40 \mathrm{~Hz}$-tACS, or active sham stimulation for 5 consecutive days. Symptom improvement was assessed using the Montgomery-Åsberg Depression Rating Scale (MADRS) as the primary outcome. High-density electroencephalograms (hdEEGs) were recorded to measure changes in alpha oscillations as the secondary outcome. For the primary outcome, we did not observe a significant interaction between treatment condition ( $10 \mathrm{~Hz}$-tACS, $40 \mathrm{~Hz}$-tACS, sham) and session (baseline to 4 weeks after completion of treatment); however, exploratory analyses show that 2 weeks after completion of the intervention, the $10 \mathrm{~Hz}$-tACS group had more responders (MADRS and HDRS) compared with $40 \mathrm{~Hz}-$ tACS and sham groups $(n=30, p=0.026)$. Concurrently, we found a significant reduction in alpha power over the left frontal regions in EEG after completion of the intervention for the group that received per-protocol $10 \mathrm{~Hz}$-tACS $(n=$ $26, p<0.05)$. Our data suggest that targeting oscillations with tACS has potential as a therapeutic intervention for treatment of MDD.
\end{abstract}

Correspondence: Flavio Fröhlich (flavio_frohlich@med.unc.edu)

${ }^{1}$ Department of Psychiatry, University of North Carolina at Chapel Hill, Chapel Hill, NC 27599, USA

${ }^{2}$ Carolina Center for Neurostimulation, University of North Carolina at Chapel Hill, Chapel Hill, NC 27599, USA

Full list of author information is available at the end of the article.

These authors contributed equally: Morgan L. Alexander and Sankaraleengam Alagapan

These authors contributed equally: David R. Rubinow and Flavio Fröhlich All data collection was completed through the Department of Psychiatry at the University of North Carolina at Chapel Hill.

\section{Introduction}

Major depressive disorder (MDD) is a common, severe psychiatric illness that has a lifetime prevalence of about $16.6 \%$ in adults ${ }^{1}$ and results in the highest burden of disability among all mental and behavioral disorders ${ }^{2}$. Current recommended drug therapies are associated with suboptimal remission rates and, oftentimes, undesirable side effects ${ }^{3}$. Furthermore, the effects of pharmacological agents are widespread; in contrast, interventions that can target specific abnormalities in brain activity may permit

\section{(c) The Author(s) 2019}

(c) (i) Open Access This article is licensed under a Creative Commons Attribution 4.0 International License, which permits use, sharing, adaptation, distribution and reproduction c. in any medium or format, as long as you give appropriate credit to the original author(s) and the source, provide a link to the Creative Commons license, and indicate if changes were made. The images or other third party material in this article are included in the article's Creative Commons license, unless indicated otherwise in a credit line to the material. If material is not included in the article's Creative Commons license and your intended use is not permitted by statutory regulation or exceeds the permitted use, you will need to obtain permission directly from the copyright holder. To view a copy of this license, visit http://creativecommons.org/licenses/by/4.0/. 
greater therapeutic precision. Patients with MDD exhibit elevated oscillatory activity, specifically in the alpha frequency band $(8-12 \mathrm{~Hz})^{4}$, which is often localized to left frontal regions, resulting in what has been called "frontal alpha asymmetry" ${ }^{\prime 5}$. Although alpha oscillations serve important functions in the healthy brain ${ }^{6,7}$, increased alpha oscillation strength in depressed patients represents a state of neuronal hypoactivity leading to disrupted affective processing ${ }^{8}$. Thus, renormalizing this elevated alpha activity could potentially mitigate symptoms of MDD. Brain activity can be altered with noninvasive brain stimulation methods such as transcranial magnetic stimulation (TMS) and transcranial electric stimulation. Rhythmic TMS bursts at the alpha frequency can entrain brain activity in healthy humans ${ }^{9}$, and synchronized TMS at individualized alpha frequencies has been evaluated for the treatment of $\mathrm{MDD}^{10}$, although TMS has not yet been demonstrated to alter alpha oscillations in these patients.

Another stimulation paradigm used to modulate endogenous brain activity is transcranial alternating current stimulation (tACS), which applies a weak electric current with a sine-wave pattern to the scalp. Such sine-wave stimulation may better lend itself to targeting oscillatory brain activity than methods such as TMS. TACS can modulate cortical oscillations that mediate cognitive function ${ }^{11}$ and can selectively modulate oscillations at the applied frequency ${ }^{12,13}$. In addition, the side effects of tACS are mild and transient, and no serious adverse events have yet been reported ${ }^{14}$. Despite these promising results, tACS has not yet been tested as a possible therapeutic intervention for MDD. To address this gap in knowledge, we hypothesized that tACS at $10 \mathrm{~Hz}$ targeting both left and right frontal areas with synchronous stimulation would improve the symptoms of MDD and restore a more physiological balance of alpha oscillations by reducing the pathologically elevated power of the alpha oscillation in left dorsolateral prefrontal cortex (dlPFC).

To test this hypothesis, we conducted a pilot double-blind study to evaluate the feasibility, safety, and efficacy of tACS as a treatment for the symptoms of depression. Patients diagnosed with MDD were randomized to one of three arms to compare $10 \mathrm{~Hz}$-tACS, $40 \mathrm{~Hz}$-tACS, and active sham stimulation. The investigation of a second, different stimulation frequency (i.e., $40 \mathrm{~Hz}$ ) served to assess whether symptom and electrophysiological changes were frequencydependent or merely stimulation-dependent. The tACS intervention comprised $40 \mathrm{~min}$ of daily stimulation for 5 consecutive days. The primary outcome was the change in Montgomery-Åsberg Depression Rating Scale (MADRS) score from baseline to the final follow-up study visit 4 weeks after completion of the intervention. To understand how tACS affects brain activity, we measured alpha power changes using high-density electroencephalography as our secondary outcome.

\section{Materials and methods}

This study was a double-blind, randomized, shamcontrolled pilot clinical trial conducted at The University of North Carolina at Chapel Hill from May 2015 to June 2017 and registered at ClinicalTrials.gov (NCT02339285). The study was approved by the Biomedical Institutional Review Board at UNC Chapel Hill (IRB \# 14-1622) and used a Data Safety Monitoring Board (DSMB) through the North Carolina Translational \& Clinical Studies Institute, to ensure participant safety. Bi-annual reviews of blinded data and adverse events were submitted to the DSMB. All participants provided written informed consent before all study-related activities.

\section{Participants}

A total of 32 patients (27 female; aged $36.69 \pm 13.08$ years) diagnosed with unipolar, non-psychotic MDD (confirmed with the M.I.N.I. International Neuropsychiatric Interview 7.0 for the Diagnostic Statistical Manual of Mental Disorders, 5th Edition), with a Hamilton Depression Rating Scale (HDRS) of $>8$ and low suicide risk, defined as scoring $<3$ on the Suicide Item on the HDRS, were randomized in this trial. Previous treatments include medication ( $94 \%$ reported) and therapy (69\% reported), indicating the enrolled participants in this sample have attempted to treat their depression before enrollment. Of the 32 enrolled participants (defined as intent-to-treat, or ITT, sample), 26 completed all study visits as designed (defined as per-protocol, or PP, sample; see CONSORT). Screened participants were excluded from participation for the following reasons: concurrent anticonvulsant medications or daily treatments with benzodiazepines (limited as-needed use that was discontinued more than $48 \mathrm{~h}$ before a study session was allowed); diagnosis of alcohol or substance dependence (other than nicotine) within the last 12 months; current Axis I mood or psychotic disorder other than MDD; lifetime comorbid psychiatric bipolar or psychotic disorder; eating disorder (current or within the past 6 months); obsessive-compulsive disorder (lifetime); posttraumatic stress disorder (current or within the last 6 months); attention-deficit/hyperactivity disorder (currently under treatment); history of significant head injury or traumatic brain injury, prior brain surgery, or any brain devices/implants; history of seizures, unstable medical illness, or pregnancy. Although not an exclusion criterion, none of the participants were left-handed (Edinburgh Handedness Inventory, $82.8 \pm 24.9)$. Screened participants were not excluded for use of antidepressants and $38 \%$ of participants were on at least one antidepressant at the time of enrollment. To control for changes in medication, participants were required to be at least 6 weeks stable on their antidepressants. See Table S1 for further baseline demographics on all randomized participants. 


\section{Study schedule}

Participants who completed the study attended a total of eight sessions (Fig. S1). Inclusion and exclusion criteria were assessed with a preliminary phone screening and then more extensively at the initial session with the study coordinator. At the initial session, participants signed consent and completed several questionnaires (demographics, Edinburgh handedness Inventory, "Hunter Beliefs About Treatment Questionnaire," used with permission of the UCLA Laboratory of Brain, Behavior, and Pharmacology, @2005, 2017 UC Regents). In addition, the study coordinator administered the M.I.N.I. and the HDRS to confirm eligibility. Before randomization, eligible participants also met with an experienced mood disorders clinician (D.R.R.) to further assess their clinical symptoms and to verify the participants met the inclusion criteria. Once eligibility was confirmed, participants returned for 5 consecutive days of treatment (Day 1 to Day 5). Baseline scores for all assessments were completed on Day 1. Participants also attended a 2-week follow-up and a 4-week follow-up after they completed the week of stimulation.

\section{Randomization}

Participants were randomized into three study arms $(10 \mathrm{~Hz}$-tACS, $n=10 ; 40 \mathrm{~Hz}$-tACS, $n=11$; and active sham at $10 \mathrm{~Hz}, n=11$ ). Intervention type was based on study codes prepared by a member of the research lab, who was not otherwise associated with the study, and codes were randomized such that no more than three participants in a row received the same intervention. All authors and members of the research team were unaware of the group assignments until completion of the entire study. To administer stimulation in a double-blind manner, we developed a custom Matlab-controlled computer interface (Mathworks, Natick, MA; NIDaq USB 6001, National Instruments, TX, USA) to control two Neuroconn DC plus stimulators (Neuroconn Ltd., Ilmenau, Germany) that delivered the stimulation based on the study code entered. To ensure that the correct waveform was applied for each session, this interface recorded the applied waveform for subsequent verification by a group member not associated with the study.

\section{Stimulation}

All three study arms used the same electrode montage (Fig. 1). Three electrodes with Ten20 paste (Bio-Medical Instruments, Clinton Township, Michigan) were applied to the scalp. Two $5 \times 5 \mathrm{~cm}$ electrodes were placed over the left and right frontal areas (F3 and F4, respectively, in the 10-20 placement system) with a third $5 \times 7 \mathrm{~cm}$ "return/ reference" electrode placed over the vertex $(\mathrm{Cz}$ in the 10-20 system). The electrode montage described here delivers in-phase synchronized stimulation to both the left and right frontal regions to target the imbalance between frontal alpha activity.

Each participant completed 5 consecutive days of the intervention (40 min of stimulation) at approximately the same time of day $( \pm 90 \mathrm{~min})$. The choice of intervention duration (5 consecutive days, $40 \mathrm{~min}$ each day) was informed by a previous study of transcranial direct current stimulation, which found efficacy 4 weeks after completion of treatment ${ }^{15}$. The tACS stimulation waveform was a sine-wave with an amplitude of $2 \mathrm{~mA}$ at $\mathrm{Cz}$ and an amplitude of $1 \mathrm{~mA}$ at F3 and F4 (amplitudes are reported as zero-to-peak). There were two tACS conditions: the proposed therapeutic frequency of $10 \mathrm{~Hz}$ and the control frequency of $40 \mathrm{~Hz}$. Previous research indicates that gamma oscillations have a stronger relationship to cognition ${ }^{16}$, and would theoretically not target alpha oscillations and not result in mood symptom changes; therefore, $40 \mathrm{~Hz}$-tACS would be an appropriate control frequency for this trial. Active sham stimulation included $20 \mathrm{~s}$ of ramp-in to $40 \mathrm{~s}$ of $10 \mathrm{~Hz}$-tACS, with a ramp-out of $20 \mathrm{~s}$, for a total of $80 \mathrm{~s}$ of stimulation. Both $10 \mathrm{~Hz}$ and $40 \mathrm{~Hz}$-tACS included $20 \mathrm{~s}$ of ramp-in to $40 \mathrm{~min}$ of stimulation, with a ramp-out of $20 \mathrm{~s}$ for a total of $2440 \mathrm{~s}$ of stimulation (Fig. 1b). During each stimulation session, participants were seated comfortably upright with their eyes open and were asked to focus on a ReefScapes video (Undersea Productions, Queensland, Australia) presented on a large projector screen directly in front of them. This video served the purpose of masking the phosphenes induced by tACS and keeping all participants in the same state during stimulation sessions. On the final day of stimulation (Day 5), participants were asked whether they believed they received stimulation over the past week (Yes, No, I don't know) to assess blinding.

\section{High-density electroencephalography}

Resting-state EEG (RSEEG) was collected at Day 1 (baseline), Day 5, and the 4-week follow-up, using a 128 channel EEG system (Geodesic EEG system 410, Electrical Geodesics, Inc., OR, USA). RSEEG was administered before the final stimulation on Day 5 to avoid recording the immediate aftereffects of tACS. Participants followed pre-programmed computer-generated instructions (Presentation, Neurobehavioral Systems, CA, USA) and had their eyes closed for $2 \mathrm{~min}$, following which they had their eyes open for $2 \mathrm{~min}^{17}$. This sequence was repeated twice resulting in a total of $8 \mathrm{~min}$ of RSEEG. The sequence was counterbalanced across participants, i.e., half of the participants started with 2 min of eyes-open condition and the other half started with 2 min of eyes-closed condition. During the eyes-open condition, participants were instructed to fixate on a cross-hair. Participants also completed a working memory task, the results of which are not presented here. 

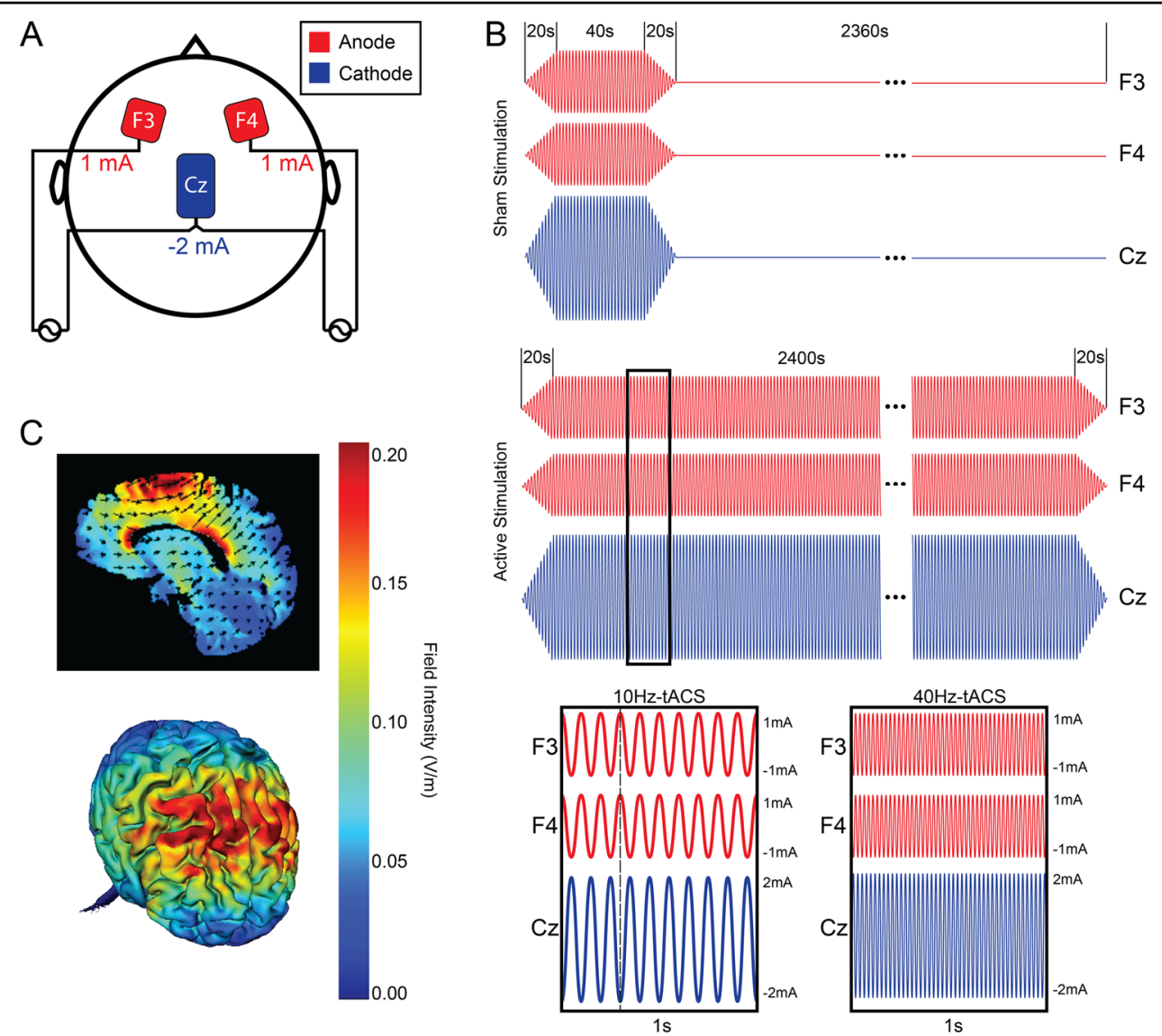

Fig. 1 a Stimulation configuration for all participants. Two stimulators were used; one connected to the electrode over F3, one connected to the electrode over F4, and both connected to the electrode over $\mathrm{Cz}$. The red electrodes ( $\mathrm{F} 3$ and F4) are the anode and the blue return electrode over $\mathrm{Cz}$ is the cathode. $\mathbf{b}$ Sham and active stimulation paradigms. Ramp-in and ramp-out is $20 \mathrm{~s}$ for all conditions, with $40 \mathrm{~s}$ of active stimulation for Sham stimulation, $2400 \mathrm{~s}$ of active stimulation for $10 \mathrm{~Hz}$-tACS and $40 \mathrm{~Hz}$-tACS. Anodes (F3 and F4) and cathode (Cz) are at opposite phase at any given point during stimulation. c Electric field simulation: 2D (top) and 3D (bottom) representation (HD-Explore, Soterix Medical, New York, NY, USA)

EEG analysis was performed using EEGLab ${ }^{18}$ and custom-written Matlab scripts. Preprocessing consisted of band-pass filtering to $1-50 \mathrm{~Hz}$, downsampling to $250 \mathrm{~Hz}$, removal of bad channels based on low correlations to surrounding channels, and artifact subspace reconstruction $^{19}$ followed by independent component analysis ${ }^{20}$ to remove artifacts caused by eye blinks, eye movements, muscle activity, and heartbeats. EEG data were separated according to the eyes-open and eyes-closed condition, and epoched into $10 \mathrm{~s}$ segments, following which power spectral density was estimated using multi-taper windowed fast Fourier transform method ${ }^{21}$. To determine changes in RSEEG at Day 5 and the 4-week follow-up, spectral power in the alpha frequency band $(8-12 \mathrm{~Hz})$ was calculated and decibel-normalized to spectral power estimated from baseline at each individual electrode as well as averages within a topographical region. The former was used to assess significant changes in oscillation power within each group, whereas the latter was used to compare changes across the three groups. In addition, alpha power in baseline session was log-transformed and compared between the groups.

\section{Side effects and safety}

Side effects were assessed after every stimulation session. Suicidal thoughts/actions were monitored daily with a selfreport questionnaire, starting from baseline until the 4week follow-up as well as during clinical assessments (MADRS and HDRS). Possible development of mania was monitored with the Young Mania Rating Scale (YMRS) administered at every study visit from baseline until the 4week follow-up. The Montreal Cognitive Assessment (MoCA) was administered at two time points (baseline, 4week follow-up) to assess any possible cognitive changes. 


\section{Outcome measures}

The primary outcome measure was defined as the change in depressive symptoms measured by the MADRS from baseline to the 4-week follow-up for the ITT sample. The MADRS was administered before stimulation at baseline, after the fifth stimulation on Day 5, at the 2-week follow-up, and the 4-week follow-up. The secondary outcome was the change in raw alpha power measured at the 4-week follow-up relative to baseline for the PP sample. The choice of the 4-week follow-up as the primary outcome was based on a previous trial using electrical stimulation ${ }^{15}$. Exploratory outcome measures were defined as the change in the HDRS and Beck Depression Inventory (BDI). Response to treatment was defined as at least a 50\% reduction in symptoms from baseline for each clinical assessment ${ }^{22}$. Remission for the MADRS was defined as scoring $\leq 9$, remission for the HDRS was defined as scoring $\leq 7$, and remission for the BDI was defined as scoring $\leq 12^{23}$.

\section{Statistical analysis}

Custom-written scripts in $\mathrm{R}$ ( $\mathrm{R}$ Foundation for Statistical Computing, Vienna, Austria) were used for analysis and are available by request. Libraries used in $\mathrm{R}$ included lme $4^{24}$ and pbkrtest $^{25}$. Differences in demographics and baseline characteristics of the three study arms and the severity of adverse effects were assessed with one-way analysis of variance (ANOVA), $\chi^{2}$-tests of independence, and pairwise $t$-test with false discovery rate (FDR) correction. Spearman's rank-order correlation was used to assess the possible role of placebo response in the effects observed using a belief of treatments questionnaire, as the data was non-parametric. To assess equality of variance, we used Bartlett's K-squared. We used a linear mixed model analysis with fixed factors of "session" (baseline, the 4-week follow-up) and "condition" (10 Hz-tACS, $40 \mathrm{~Hz}$ tACS, active sham $10 \mathrm{~Hz}$-tACS), with random factor "participant" to account for repeated measures within participants. The choice of a linear mixed model for the main outcome takes into consideration any missing data in our ITT sample. The interaction between "session" and "condition" is defined as the effects of "session" on "condition". Kenward-Roger approximations were used to calculate $p$-values and perform F-tests for each factor and their interaction in the mixed model. Effect sizes between groups were calculated using eta-squared $\left(\eta^{2}\right)$, and effect sizes within groups (baseline to Day 5, 2-week follow-up, 4-week follow-up) were calculated using Cohen's $d$. Differences in rates of response and remission of the three study arms and the success of blinding to treatment arm were assessed with $\chi^{2}$-tests of independence.

Statistical analysis of EEG data was performed using custom R scripts and the "lmertest" package ${ }^{26}$, which allows fitting linear mixed-effects model and uses Satterthwaite's approximation to degrees of freedom, to determine the F statistics of the fixed effects. Alpha power changes were averaged across electrodes over different topographical regions (frontal, central, occipital, temporal/parietal; Fig. S2A). Alpha asymmetry was measured as $\ln \left(\frac{\text { right alpha power }}{\text { left alpha power }}\right)$ of the pooled average of the left and right frontal electrodes. Linear mixed-effects models were fit with power change as the dependent variable, topographical region and condition as fixed factors and participant as random factor. The residuals of the models were tested for normality using the Shapiro-Wilk test. Two different models were fit for Day 5 and the 4-week follow-up. Post-hoc analysis was performed to get contrasts adjusted using Tukey's honest significant difference (HSD) in the "emmeans" package. Baseline alpha power differences were determined by fitting a linear model with log-transformed baseline alpha power as a dependent variable, and condition and region as factors. To determine which electrodes exhibited significant power change, we performed a one-sample $t$-test with FDR correction. Spearman's correlation coefficient was computed between log-transformed baseline alpha scores and changes.

\section{Sample size determination}

The target sample size was 30 participants, with $n=10$ for each arm of the study. This sample was chosen based on funding duration and a focus on feasibility, as this was the first study to use tACS in this population; however, several studies of tACS in healthy populations used a similar sample size to show changes in alpha ${ }^{27,28}$. A total of 32 participants were randomized (ITT sample), with 26 completing all study sessions (PP sample). Enrollment ended because funding had ended.

\section{Code availability}

All codes used to analyze the presented results are available upon request.

\section{Results \\ Primary outcome (MADRS)}

In the ITT analysis, MADRS scores for all three groups decreased significantly from baseline to the 4-week follow-up, but there was no significant difference in these changes based upon condition $(10 \mathrm{~Hz}$-tACS, $40 \mathrm{~Hz}$-tACS, active sham $10 \mathrm{~Hz}$-tACS) (i.e., there was a significant effect of session $\left(\mathrm{F}_{1,28.618}=38.87, p<0.001\right)$, but not condition $\left(\mathrm{F}_{2,28.681}=0.22, \quad p=0.80\right) \quad$ or interaction $\left.\left(\mathrm{F}_{2,26.559}=0.65, p=0.53\right)\right)$.

\section{Secondary outcome (EEG)}

To verify whether tACS was effective in engaging alpha oscillations, we assessed changes in resting-state alpha 


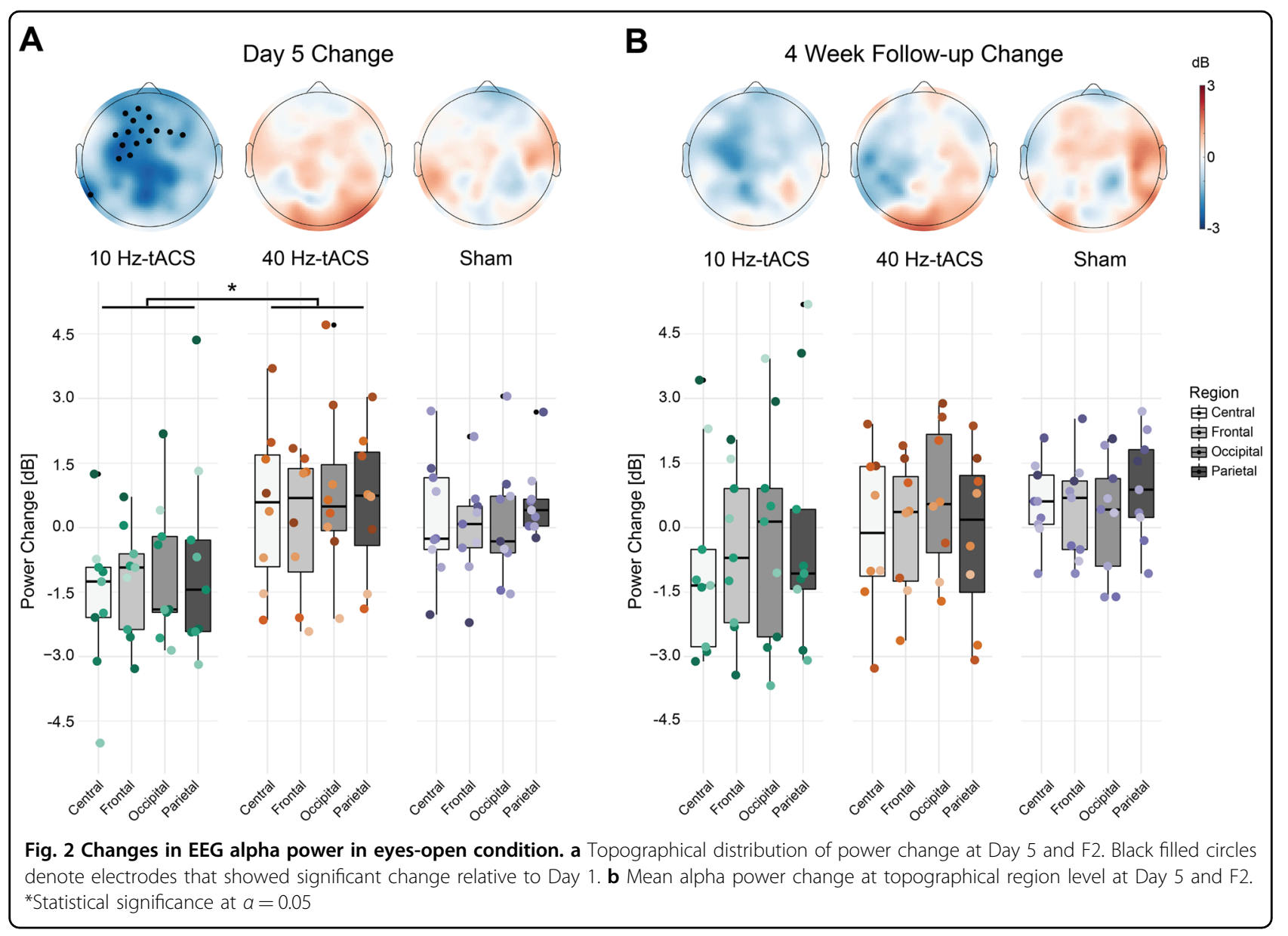

power at Day 5 (Fig. 2a) and the 4-week follow-up (Fig. 2b) in the PP sample. We performed statistical analysis of alpha power change at individual electrode level and at region level. Baseline alpha power was not different between the three groups (Fig. S2C; linear model factor condition $\mathrm{F}_{2,92}=0.126, p=0.881$; factor region $\mathrm{F}_{3,92}=3.727, p=0.014$, interaction $\mathrm{F}_{6,92}=0.042, p=$ $0.99)$ nor did we find significant alpha asymmetry in our participant sample $(0.0199 \pm 0.0336$ (mean \pm SEM), $p=$ 0.5584 one-sample $t$-test) or within any treatment groups $(10 \mathrm{~Hz}$-tACS: $-0.0040 \pm 0.0592, p=0.9474 ; 40 \mathrm{~Hz}$-tACS: $0.0585 \pm 0.0548, p=0.3211$; sham: $0.0096 \pm 0.0639, p=$ 0.8847). Post-hoc analysis of contrasts in factor region revealed that the difference between frontal and occipital regions $(p=0.020)$ and the difference between occipital and parietal regions were significant $(p=0.039)$, whereas the difference between central and occipital regions were trend-level $(p=0.087)$. At individual electrode level, the $10 \mathrm{~Hz}$-tACS group showed significant decrease over the left frontal regions (black circles in Fig. 2a, $p<0.05$, onesample $t$-test with FDR correction), whereas no effect of stimulation was observed in the other groups at Day 5 or in any of the groups at the 4-week follow-up. We did not find any significant differences between the three stimulation conditions on frontal alpha asymmetry on Day 5 relative to Day $1 \quad\left(\mathrm{~F}_{2,23}=0.24, p=0.7852\right.$ one-way ANOVA) nor did we find any significant effect of stimulation on Day 5 relative to Day 1 within any treatment groups $(10 \mathrm{~Hz}-\mathrm{tACS}: 0.0144 \pm 0.0330, p=0.6738$; $40 \mathrm{~Hz}$ tACS: $0.0145 \pm 0.0246, \quad p=0.5739$; sham: $-0.0171 \pm$ 0.0477, $p=0.7289$ ). We observed significant negative correlations between the log-transformed baseline alpha power and alpha power changes at Day 5 in all the regions (Fig. S2B; Spearman's rank correlations, Frontal $\rho=$ $-0.45, p=0.02$; Central $\rho=-0.54, p<0.01$; Parietal $\rho=$ $-0.65, p<0.01$; Occipital $\rho=-0.55, p<0.01)$. Thus, we included the log-transformed baseline alpha power as a covariate in our analysis of the effects of the intervention. To assess the effect of stimulation on alpha power on Day 5 , we fitted a linear mixed model with power change in alpha band as dependent variable and condition (3 levels $-10 \mathrm{~Hz}-\mathrm{tACS}, 40 \mathrm{~Hz}$-tACS, and Sham) and regions (4 levels-frontal, central, parietal, and occipital) as fixed factors and participants as random factors. ANOVA of the model revealed significant effect of condition $\left(\mathrm{F}_{2,21.595}=\right.$ 3.931, $p=0.035)$ and region $\left(\mathrm{F}_{3,79.358}=7.762, p<0.001\right)$. 
There was no significant effect of interaction $\left(\mathrm{F}_{6,68.284}=\right.$ 1.073, $p=0.388$ ). Post-hoc analysis of contrasts in factor condition using Tukey's HSD revealed significant difference between $10 \mathrm{~Hz}$-tACS group and $40 \mathrm{~Hz}$-tACS group $(p=0.039)$. For factor region, significant differences were found between frontal and occipital $(p<0.001)$, central and occipital $(p<0.01)$, and frontal and parietal $(p<0.05)$, whereas the difference between occipital and parietal was trend-level $(p<0.1)$. ANOVA of the linear mixed model for alpha power changes at the 4-week follow-up revealed significant effect of only region $\left(\mathrm{F}_{3,80.094}=2.921, p=\right.$ 0.039 ). There was no significant effect of condition $\left(\mathrm{F}_{2,22.722}=0.629, p=0.542\right)$, whereas interaction was trend-level $\left(\mathrm{F}_{6,69.170}=2.019, p=0.075\right)$. Post-hoc analysis revealed that the differences between frontal and occipital, and central and occipital were trend-level $(p<0.1)$. At individual electrode level, the $10 \mathrm{~Hz}$-tACS group showed significant decrease from baseline to Day 5 over the left frontal regions (black circles in Fig. 2a, $p<0.05$, onesample $t$-test with FDR correction), whereas no effect of stimulation was observed in the other groups at Day 5 or in any of the groups at the 4-week follow-up. There was no statistically significant correlation between change in alpha asymmetry and change in MADRS $(\rho=0.0316, p=$ 0.8784 ) nor between change in frontal alpha oscillations and change in MADRS $(\rho=0.1865 ; p=0.6397)$. Taken together, our results indicate that $10 \mathrm{~Hz}$-tACS was effective in targeting alpha oscillations predominantly in the frontal and central regions, but this change did not have a relationship with change in clinical symptoms. Similar analysis of eyes-closed data did not reveal any significant effect of stimulation in alpha power change.

\section{Response and remission (MADRS, HDRS, BDI)}

Within the ITT sample, we found a significant relationship between condition and response rates for the MADRS $\left(x^{2}=7.334, \mathrm{df}=2, p=0.026\right)$ and HDRS $\left(x^{2}=\right.$ $7.334, \mathrm{df}=2, p=0.026)$ at the 2-week follow-up. This indicates that the $10 \mathrm{~Hz}$-tACS group had a higher rate of response at the 2-week follow-up (77.8\%) than the $40 \mathrm{~Hz}$ tACS $(30.0 \%)$ and sham stimulation groups (20.0\%) (Fig. 3). No other significant relationships were found for response rates (Table 1). We also looked into remission rates over the course of the intervention; however, around half of participants did not qualify for remission at any time point as measured by the MADRS (59\% did not remit), the HDRS (50\% did not remit), and the BDI (41\% did not remit). No significant relationships were found for remission rates (Table 1).

\section{Effect sizes (MADRS, HDRS, BDI)}

In exploratory analyses, we calculated effect sizes between and within the treatment groups in the ITT sample. We looked at the mean (SD) change in MADRS from baseline to Day 5, the 2-week follow-up, and the 4week follow-up (Table S2). The change in scores from baseline to the 4-week follow-up demonstrated a moderate effect size between conditions $\left(\eta^{2}=0.060\right)$ and the largest effect size was seen in the change from baseline to the 2-week follow-up $\left(\eta^{2}=0.116\right)$. We also calculated Cohen's $d$ in each treatment group from baseline to Day 5, the 2-week follow-up, and the 4-week follow-up. For the MADRS, the largest within-group effect size was seen in the $10 \mathrm{~Hz}$-tACS group from baseline to the 2-week follow-up $(d=1.70)$, with the next largest seen in the $10 \mathrm{~Hz}$-tACS group from baseline to the 4-week follow-up $(d=1.60)$.

We next sought to validate the effect of tACS on improving MDD symptoms by including analogous rating scales. The HDRS was used as a complementary outcome measure to the MADRS and the scores were strongly correlated $\left(R^{2}=0.85, p<0.001\right)$. The mean (SD) change in HDRS from baseline to the 4-week follow-up was -8.67 (5.32) for $10 \mathrm{~Hz}$-tACS, -5.20 (5.67) for $40 \mathrm{~Hz}$-tACS, and -5.11 (7.61) for sham stimulation. Similar to the MADRS, this change demonstrated a moderate between-group effect size $\left(\eta^{2}=0.071\right)$. For the HDRS, the largest within-group effect size was seen in the $10 \mathrm{~Hz}$-tACS group from baseline to the 4-week follow-up $(d=1.61)$, with the next largest seen in the $10 \mathrm{~Hz}$-tACS group from baseline to the 2-week followup $(d=1.58)$.

In addition, the BDI was collected as a self-report measure of symptom changes and was strongly correlated with the MADRS $\left(R^{2}=0.72, p<0.001\right)$. The mean (SD) change in BDI from baseline to the 4-week follow-up was -14.78 (13.14) for $10 \mathrm{~Hz}-\mathrm{tACS},-12.20(11.68)$ for $40 \mathrm{~Hz}-$ tACS, and -12.33 (10.71) for sham stimulation. This change demonstrated a small between-group effect size $\left(\eta^{2}=0.011\right)$. For the BDI, the largest within-group effect size was seen in the $10 \mathrm{~Hz}$-tACS group at the 4-week follow-up $(d=1.54)$, with the next largest seen in the sham group from baseline to the 2-week follow-up $(d=1.44)$.

\section{Safety}

We used one-way ANOVAs to determine group differences in the experience and expectations of side effects (Table S3). Participants from all three groups reported minimal side effects and there was no difference between the three groups with the exception of "flickering lights" (or phosphenes, $p=0.014$ ). We ran post-hoc paired $t$ tests with FDR correction and found that there was not a significant difference in this side effect between $10 \mathrm{~Hz}$ tACS and $40 \mathrm{~Hz}$-tACS $(p=0.99)$; however, there was a trend-level difference between $10 \mathrm{~Hz}$-tACS and sham $(p=0.09)$, and a significant difference between $40 \mathrm{~Hz}-$ tACS and sham $(p<0.01)$. 


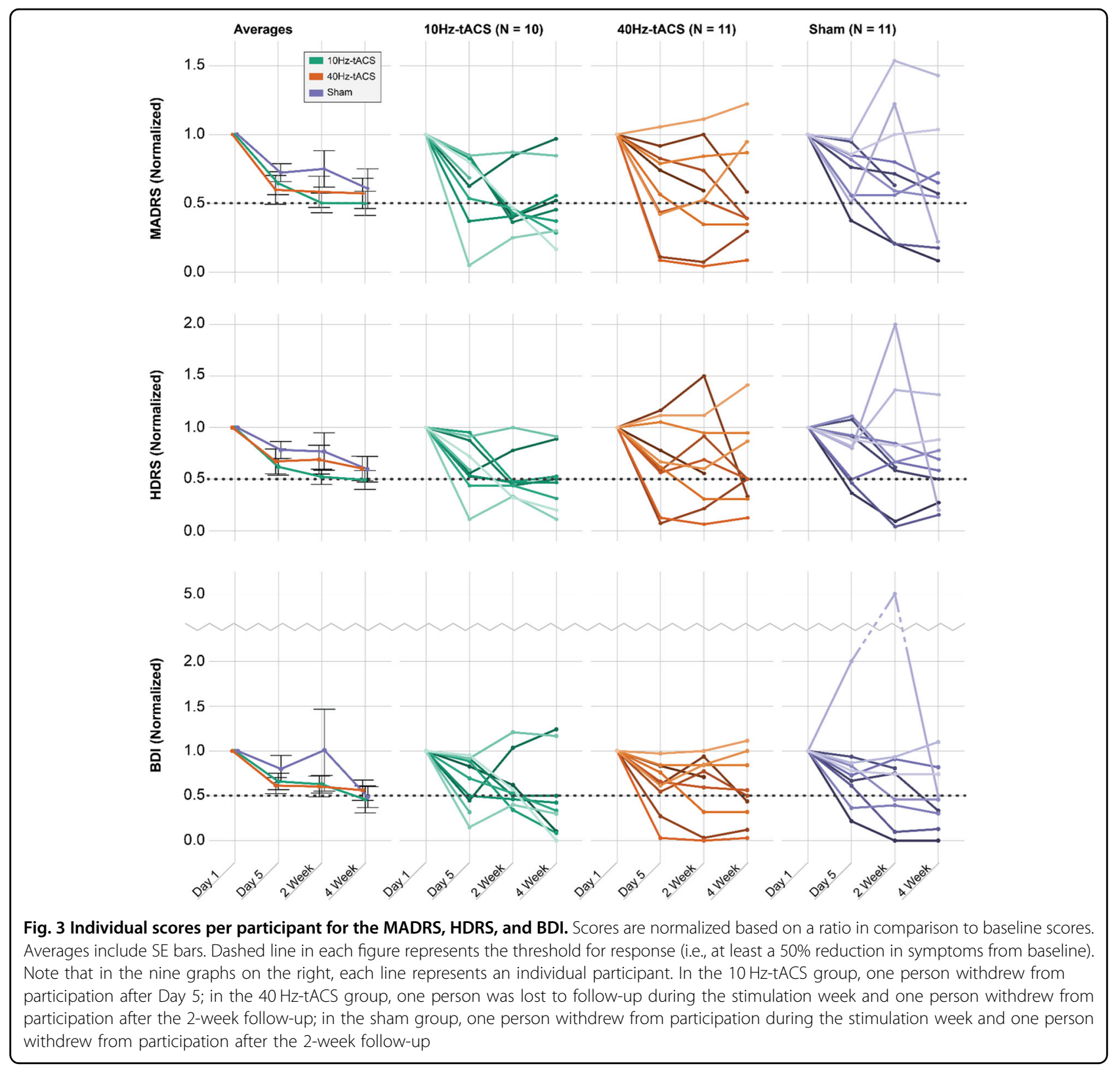

After enrollment, four participants in the sham stimulation group experienced an increase in suicidal ideation and one of these four participants reported suicidal plans (i.e., scoring $>3$ on the suicide item on the HDRS). No participants in the $10 \mathrm{~Hz}$-tACS and $40 \mathrm{~Hz}$-tACS groups experienced an increase in suicidal ideation from baseline during the course of the study.

No participants developed mania or hypomania at any point during study participation based on the YMRS. Two adverse events were reported during the course of the study; however, after a thorough investigation, neither were determined to be related to the study or intervention. Finally, participants from all three groups experienced a small improvement in cognition from baseline to the 4-week follow-up as measured by the MoCA (Table S2). There was no significant difference in this improvement in relation to condition across sessions $\left(\mathrm{F}_{2,25.717}=1.354, p=0.276\right)$.

\section{Blinding}

Self-report responses from participants on whether they thought they received verum stimulation (Yes, No, I don't know) and the treatment group $(10 \mathrm{~Hz}-\mathrm{tACS}, 40 \mathrm{~Hz}-$ tACS, sham) were significantly related $\left(\chi^{2}=8.304, \mathrm{df}=2\right.$, $p=0.016)$. Those who received $40 \mathrm{~Hz}$-tACS were more likely to think they had been stimulated, with $90 \%$ correctly reporting that they received stimulation. For those who received $10 \mathrm{~Hz}-\mathrm{tACS}, 40 \%$ reported that they 
Table 1 Response (the number of participants that achieved at least a 50\% reduction in symptoms compared with baseline) and remission rates at each time for each assessment and each time point

\begin{tabular}{|c|c|c|c|c|c|c|}
\hline & \multicolumn{2}{|c|}{ MADRS, $n(\%)$} & \multicolumn{2}{|c|}{ HDRS, $n$ (\%) } & \multicolumn{2}{|l|}{ BDI, $n$ (\%) } \\
\hline & Response & Remission & Response & Remission & Response & Remission \\
\hline \multicolumn{7}{|l|}{ Day 5} \\
\hline $10 \mathrm{~Hz}$-tACS $(n=10)$ & $2(20.0)$ & $1(10.0)$ & $2(20.0)$ & $2(20.0)$ & $4(40.0)$ & $2(20.0)$ \\
\hline $40 \mathrm{~Hz}-\mathrm{tACS}(n=10)$ & $4(40.0)$ & $3(30.0)$ & $2(20.0)$ & $4(40.0)$ & $2(20.0)$ & $5(50.0)$ \\
\hline Sham $(n=10)$ & $2(20.0)$ & $2(20.0)$ & $3(30.0)$ & $3(30.0)$ & $2(20.0)$ & $3(30.0)$ \\
\hline$x^{2}$ & 1.364 & 1.25 & 0.373 & 0.953 & 1.364 & 2.100 \\
\hline$d f$ & 2 & 2 & 2 & 2 & 2 & 2 \\
\hline$p$ & 0.506 & 0.535 & 0.83 & 0.621 & 0.506 & 0.350 \\
\hline \multicolumn{7}{|l|}{ 2-Week follow-up } \\
\hline $10 \mathrm{~Hz}-\mathrm{tACS}(n=9)$ & $7(77.8)$ & $1(11.1)$ & $7(77.8)$ & $4(44.4)$ & $4(44.4)$ & $5(55.6)$ \\
\hline $40 \mathrm{~Hz}-\mathrm{tACS}(n=10)$ & $3(30.0)$ & $3(30.0)$ & $3(30.0)$ & $3(30.0)$ & $3(30.0)$ & $4(40.0)$ \\
\hline Sham $(n=10)$ & $2(20.0)$ & $2(20.0)$ & $2(20.0)$ & $4(40.0)$ & $4(40.0)$ & $4(40.0)$ \\
\hline$x^{2}$ & 7.334 & 1.034 & 7.334 & 0.448 & 0.448 & 0.607 \\
\hline$d f$ & 2 & 2 & 2 & 2 & 2 & 2 \\
\hline$p$ & $0.026^{*}$ & 0.596 & $0.026^{*}$ & 0.800 & 0.800 & 0.738 \\
\hline \multicolumn{7}{|l|}{ 4-Week follow-up } \\
\hline $10 \mathrm{~Hz}-\mathrm{tACS}(n=9)$ & $5(55.6)$ & $3(33.3)$ & $5(55.5)$ & $4(44.4)$ & $7(77.8)$ & $7(77.8)$ \\
\hline $40 \mathrm{~Hz}-\mathrm{tACS}(n=10)$ & $5(50.0)$ & $6(60.0)$ & $6(60.0)$ & $5(50.0)$ & $5(50.0)$ & $5(50.0)$ \\
\hline Sham $(n=9)$ & $3(33.3)$ & $3(33.3)$ & $4(44.4)$ & $6(66.7)$ & $6(66.7)$ & $6(66.7)$ \\
\hline$x^{2}$ & 0.973 & 1.867 & 0.482 & 0.973 & 1.625 & 1.625 \\
\hline$d f$ & 2 & 2 & 2 & 2 & 2 & 2 \\
\hline$p$ & 0.615 & 0.393 & 0.786 & 0.615 & 0.444 & 0.444 \\
\hline
\end{tabular}

Note that the MADRS and HDRS assess symptoms from the past week, whereas BDI assesses symptoms from the past 2 weeks. Each clinical assessment has different requirements for remission: remission for the MADRS is 9 or less, remission for the HDRS is 7 or less, and remission for the BDI is 12 or less. + denotes $p<0.10$ and * denotes $p<0.05$

received stimulation; for those who received sham stimulation, $30 \%$ reported that they had received stimulation.

\section{Expectations of treatment}

We ran one-way ANOVAs on expectations of treatment (Item 1: expected likelihood of symptom improvement; Item 2: expected improvement in symptoms) to assess whether there were group differences in these expectations of treatment, and there were not $(p=0.409, p=$ 0.990 , respectively). There was no significant relationship between either of these items and the percent change in MADRS score from baseline to the 2-week follow-up (Item 1: $\rho=0.343, p=0.054$; Item 2: $\rho=0.317, p=0.078$ ) and from baseline to the 4-week follow-up (Item 1: $\rho=$ $0.273, p=0.131$; Item 2: $\rho=0.243, p=0.181$ ). Post-hoc analysis shows that the trend-level relationship between expectations and percent change in MADRS from baseline to the 2-week follow-up was driven by the sham group (Item 1: $\rho=0.778, p=0.005$; Item 2: $\rho=0.693$, $p=0.018$ ), but not the $10 \mathrm{~Hz}$-tACS (Item $1: \rho=-0.072$, $p=0.843$; Item 2: $\rho=0.114, p=0.753)$ or $40 \mathrm{~Hz}$-tACS groups (Item 1: $\rho=0.363, p=0.272$; Item 2: $\rho=0.080$, $p=0.816)$.

\section{Discussion}

This pilot clinical trial was designed to evaluate the feasibility, safety, and preliminary efficacy of tACS as a treatment for the symptoms of MDD. Although our primary outcome measure, change in MADRS at the 4-week follow-up in the ITT sample, was not significantly different between the groups, $10 \mathrm{~Hz}$-tACS significantly outperformed $40 \mathrm{~Hz}$-tACS and sham stimulation in terms of response rates at the 2-week follow-up. These results were consistent in both clinician-administered measures (MADRS, HDRS). In addition, $10 \mathrm{~Hz}$-tACS was also 
effective in engaging alpha oscillations compared with sham or $40 \mathrm{~Hz}$-tACS. Taken together, our results suggest that targeting alpha oscillations with tACS is a potentially viable therapeutic approach and a fully powered subsequent study is justified to further establish tACS as a treatment for depression.

Stimulation was tolerated well by the participants, with no serious adverse events related to the stimulation reported and no development of mania, hypomania, or increase in suicidal ideation as a result of stimulation. Retention rates were high for all three groups. These data indicate that future trials using tACS are feasible in this population.

Our physiological target in this study was frontal alpha oscillations. Altered alpha oscillations are thought to be a key physiological marker in patients with MDD. Resting state recordings in patients with MDD are characterized by increased synchrony in the theta and alpha frequency bands ${ }^{4,29}$, the latter of which likely emerges from abnormal thalamocortical connectivity ${ }^{30}$. In addition, alpha activity in the left frontal regions has been shown to be higher than that of the right frontal regions ${ }^{5,31,32}$. This increased, asymmetric alpha activity is thought to reflect reduced neuronal activity in the left dIPFC, one of several key regions identified as abnormal in brain imaging studies of depression ${ }^{8,33}$. Although repetitive TMS (rTMS) studies for treating depression often use stimulation frequencies around the alpha band ${ }^{34}$, the effect of rTMS on oscillations in patients with MDD has seldom been demonstrated, except in a few case studies ${ }^{35,36}$. The $10 \mathrm{~Hz}$-tACS led to significantly reduced alpha oscillations over the left frontal regions along with the highest response rates, suggesting that successful reshaping of disrupted oscillations may have led to the decreased symptoms observed, despite the fact that alpha asymmetry was not observed in our sample. This indicates that $10 \mathrm{~Hz}$-tACS may have a therapeutic effect regardless of asymmetry. However, our interpretation of the results is limited by the fact that we did not collect RSEEG data at the 2-week follow-up. In studies with healthy volunteers, tACS has often been suggested to increase alpha power immediately after stimulation with effects lasting up to $70 \mathrm{~min}^{27,28,37}$. In contrast, our results indicate a decrease in alpha power. The differences could be attributed to dosage (five sessions of 40 min stimulation vs. one session of 20 min stimulation). Alternatively, the differences could be due to the altered network oscillations in patients with MDD (i.e., the impact of tACS may differ in the presence of abnormal alpha activity). The exact mechanism of tACS has not yet been determined. Studies suggest tACS could induce entrainment of cortical oscillations or plasticity (or likely a combination of both) $)^{12,28,38}$. The effects of periodic perturbation have been shown to be statedependent, further confounding the possible mechanism $^{27,39,40}$. Although the immediate after-effect of tACS may be enhancement in alpha power, repeated application of tACS may lead to a resetting of oscillators potentially through homeostatic mechanisms resulting in a decrease in alpha power, as is proposed for rTMS $^{41}$. Further studies are required to determine the mechanisms underlying the effect of tACS in patients with aberrant network oscillations.

Despite the promising results as measured by clinician-administered assessments (MADRS, HDRS), we did not see the same results in the self-report measure of the BDI. This may be due to the different time frame (past week vs. past 2 weeks) or that patients may have a delay in recognizing symptom changes compared to clinicians.

This study has several limitations. First, this was an exploratory pilot study with a small sample size, powered to detect only large effect sizes. Although we determined our sample sizes based on previous tACS results, our study investigated effects at a longer time frame compared with the previous studies. Second, blinding was not successful in the $40 \mathrm{~Hz}$-tACS group; however, the blinding was successful in the $10 \mathrm{~Hz}$-tACS group, indicating that, at least in this group, the change in symptoms may be more related to the stimulation and less likely a placebo effect. Third, further studies with EEG data collection before and after each stimulation session will be required to strengthen the evidence for alpha oscillations as the target for the $10 \mathrm{~Hz}$-tACS intervention. Finally, as this was a small pilot study, the heterogeneity introduced by medication, therapy, duration of illness, etc., may have affected our results.

To our knowledge, this is the first time tACS has been studied for the treatment of MDD, demonstrating that it may be a feasible and efficacious treatment in this population. With these results, the next steps would be to validate these results in a larger sample that can establish the use of tACS to treat MDD. Future directions may also include investigating dosage, maintenance treatment, and following participants for longer than 4 weeks after treatment.

\footnotetext{
Acknowledgements

We thank Kristin Sellers and Zhe Charles Zhou for their help with participant randomization for this study, as well as Sangtae Ahn for his guidance in the EEG analysis. The research reported in this publication was partially supported by the Brain \& Behavior Resesarch Foundation (to F.F., Award 22007). This work was also partially supported by UNC Psychiatry, UNC School of Medicine (to F.F.), the Foundation of Hope (to D.R.R. and F.F.), the Swiss National Science Foundation (to C.L., grant P300PA_164693), and by the Clinical and Translational Science Award program of the National Center for Advancing Translational Sciences, National Institutes of Health (Award 1UL1TR001111). The work presented here is further supported by the National Institute of Mental Health and the National Institutes of Health (award R01MH101547, to F.F.). The content is solely the responsibility of the authors and does not necessarily represent the official views of the National Institutes of Health.
} 


\section{Author details}

'Department of Psychiatry, University of North Carolina at Chapel Hill, Chapel Hill, NC 27599, USA. ${ }^{2}$ Carolina Center for Neurostimulation, University of North Carolina at Chapel Hill, Chapel Hill, NC 27599, USA. ${ }^{3}$ Neural Control of Movement Lab, Department of Health Sciences and Technology, ETH Zurich, Zurich 8092, Switzerland. ${ }^{4}$ Department of Neurology, University of North Carolina at Chapel Hill, Chapel Hill, NC 27599, USA. ${ }^{5}$ Department of Biomedical Engineering, University of North Carolina at Chapel Hill, Chapel Hill, NC 27599, USA. ${ }^{6}$ Department of Cell Biology and Physiology, University of North Carolina at Chapel Hill, Chapel Hill, NC 27599, USA. ${ }^{7}$ Neuroscience Center, University of North Carolina at Chapel Hill, Chapel Hill, NC 27599, USA

\section{Conflict of interest}

F.F. is the lead inventor of an IP filed by UNC. The clinical studies performed in the Frohlich Lab have originally received a designation as conflict of interest with administrative considerations that was subsequently removed. F.F. is the founder, CSO, and majority owner of Pulvinar Neuro LLC. The company had no role in this study. F.F. is also an author under Elsevier and receives royalty payments. D.R.R. reports consulting fees, travel reimbursement, and stock options as a member of the Clinical Advisory Board of Sage Therapeutics. D.R.R. also received compensation as a member of the Editorial Board of Dialogues in Clinical Neurosciences (Servier). All other authors declare that they have no conflict of interest.

\section{Publisher's note}

Springer Nature remains neutral with regard to jurisdictional claims in published maps and institutional affiliations.

Supplementary information accompanies this paper at (https://doi.org/ 10.1038/s41398-019-0439-0).

Received: 29 September 2018 Revised: 11 February 2019 Accepted: 13 February 2019

Published online: 05 March 2019

\section{References}

1. Kessler, R. C. et al. Lifetime prevalence and age-of-onset distributions of DSMIV disorders in the National Comorbidity Survey Replication. Arch. Gen. Psychiatry 62, 593-602 (2005).

2. Murray, C. J. et al. The state of US health, 1990-2010: burden of diseases, injuries, and risk factors. JAMA 310, 591-608 (2013).

3. DeRubeis, R. J., Siegle, G. J. \& Hollon, S. D. Cognitive therapy versus medication for depression: treatment outcomes and neural mechanisms. Nat. Rev. Neurosci. 9, 788-796 (2008).

4. Leuchter, A. F., Cook, I. A., Hunter, A. M., Cai, C. \& Horvath, S. Resting-state quantitative electroencephalography reveals increased neurophysiologic connectivity in depression. PLOS ONE 7, e32508 (2012).

5. Henriques, J. B. \& Davidson, R. J. Regional brain electrical asymmetries discriminate between previously depressed and healthy control subjects. J. Abnorm. Psychol. 99, 22-31 (1990).

6. Klimesch, W. alpha-band oscillations, attention, and controlled access to stored information. Trends Cogn. Sci. 16, 606-617 (2012).

7. Jensen, O. \& Mazaheri, A. Shaping functional architecture by oscillatory alpha activity: gating by inhibition. Front. Hum. Neurosci. 4, 186 (2010).

8. Galynker, I. I., Cai, J., Ongseng, F., Finestone, H., Dutta, E. \& Serseni, D. Hypofrontality and negative symptoms in major depressive disorder. J. Nud. Med. 39, 608-612 (1998).

9. Thut, G. et al. Rhythmic TMS causes local entrainment of natural oscillatory signatures. Curr. Biol. 21, 1176-1185 (2011).

10. Leuchter, A. F. et al. Efficacy and safety of low-field synchronized transcranial magnetic stimulation (sTMS) for treatment of major depression. Brain Stimul. 8, 787-794 (2015).

11. Kasten, F. H. \& Herrmann, C. S. Transcranial alternating current stimulation (tACS) enhances mental rotation performance during and after stimulation. Front. Human. Neurosci. 11, 2 (2017).
12. Ali, M. M., Sellers, K. K. \& Frohlich, F. Transcranial alternating current stimulation modulates large-scale cortical network activity by network resonance. J. Neurosci. 33, 11262-11275 (2013).

13. Herrmann, C. S., Rach, S., Neuling, T. \& Struber, D. Transcranial alternating current stimulation: a review of the underlying mechanisms and modulation of cognitive processes. Front Hum. Neurosci. 7, 279 (2013).

14. Antal A., et al. Low intensity transcranial electric stimulation: Safety, ethical, legal regulatory and application guidelines. Clin. Neurophysiol. 128, 1774-1809 (2017).

15. Brunoni, A. R. et al. The sertraline vs. electrical current therapy for treating depression clinical study: results from a factorial, randomized, controlled trial. JAMA Psychiatry 70, 383-391 (2013).

16. Miyaguchi, S. et al. Transcranial alternating current stimulation with gamma oscillations over the primary motor cortex and cerebellar hemisphere improved visuomotor performance. Front. Behav. Neurosci. 12, 132 (2018).

17. Barry, R. J., Clarke, A. R., Johnstone, S. J., Magee, C. A. \& Rushby, J. A. EEG differences between eyes-closed and eyes-open resting conditions. Clin. Neurophysiol. 118, 2765-2773 (2007).

18. Delorme, A. \& Makeig, S. EEGLAB: an open source toolbox for analysis of single-trial EEG dynamics including independent component analysis. J. Neurosci. Methods 134, 9-21 (2004).

19. Mullen, T. et al. Real-time modeling and 3D visualization of source dynamics and connectivity using wearable EEG. Conf. Proc. IEEE Eng. Med. Biol. Soc. 2013, 2184-2187 (2013).

20. Jung, T. P. et al. Removing electroencephalographic artifacts by blind source separation. Psychophysiology 37, 163-178 (2000).

21. Bokil, H., Andrews, P., Kulkarni, J. E., Mehta, S. \& Mitra, P. P. Chronux: a platform for analyzing neural signals. J. Neurosci. Methods 192, 146-151 (2010).

22. Zimmerman, M., Posternak, M. A. \& Chelminski, I. Defining remission on the Montgomery-Asberg depression rating scale. J. Clin. Psychiatry 65, 163-168 (2004).

23. Riedel, $\mathbf{M}$. et al. Response and remission criteria in major depression--a validation of current practice. J. Psychiatr. Res. 44, 1063-1068 (2010).

24. Bates, D., Machler, M., Bolker, B. M. \& Walker, S. C. Fitting linear mixed-effects models using Ime4. J. Stat. Softw. 67, 1-48 (2015).

25. Halekoh, U. \& Hojsgaard, S. Kenward-Roger approximation and parametric bootstrap methods for tests in linear mixed models - The R Package pbkrtest. J. Stat. Softw. 59, 1-32 (2014).

26. Kuznetsova, A., Brockhoff, P. B. \& Christensen, R. H. B. ImerTest package: tests in linear mixed effects models. J. Stat. Softw. 82, 1-26 (2017).

27. Neuling, T., Rach, S. \& Herrmann, C. S. Orchestrating neuronal networks: sustained after-effects of transcranial alternating current stimulation depend upon brain states. Front. Hum. Neurosci. 7, 161 (2013).

28. Vossen, A., Gross, J. \& Thut, G. Alpha power increase after transcranial alternating current stimulation at alpha frequency (alpha-tACS) reflects plastic changes rather than entrainment. Brain Stimul. 8, 499-508 (2015).

29. Fingelkurts, A. A. et al. Impaired functional connectivity at EEG alpha and theta frequency bands in major depression. Hum. Brain Mapp. 28, 247-261 (2007).

30. Greicius, M. D. et al. Resting-state functional connectivity in major depression: abnormally increased contributions from subgenual cingulate cortex and thalamus. Biol. Psychiatry 62, 429-437 (2007).

31. Stewart, J. L., Coan, J. A., Towers, D. N. \& Allen, J. J. B. Resting and task-elicited prefrontal EEG alpha asymmetry in depression: support for the capability model. Psychophysiology 51, 446-455 (2014).

32. Allen, J. J. B. \& Reznik, S. J. Frontal EEG asymmetry as a promising marker of depression vulnerability: summary and methodological considerations. Curr. Opin. Psychol. 4, 93-97 (2015).

33. Koenigs, M. \& Grafman, J. The functional neuroanatomy of depression: distinct roles for ventromedial and dorsolateral prefrontal cortex. Behav. brain Res. 201, 239-243 (2009).

34. Perera, T. et al. The Clinical TMS Society consensus teview and treatment recommendations for TMS therapy for major depressive disorder. Brain Stimul. 9, 336-346 (2016)

35. Pellicciari, M. C., Ponzo, V., Caltagirone, C. \& Koch, G. Restored asymmetry of prefrontal cortical oscillatory activity after bilateral theta burst stimulation treatment in a patient with major depressive disorder: a TMS-EEG study. Brain Stimul. 10, 147-149 (2017).

36. Vanneste, S., Ost, J., Langguth, B. \& De Ridder, D. TMS by double-cone coil prefrontal stimulation for medication resistant chronic depression: a case report. Neurocase 20, 61-68 (2014). 
37. Kasten F. H., Dowsett J., Herrmann C. S. Sustained aftereffect of alpha-tACS lasts up to 70 min after stimulation. Front. Hum. Neurosci. 10, 245 (2016).

38. Helfrich, R. F. et al. Entrainment of brain oscillations by transcranial alternating current stimulation. Curr. Biol. 24, 333-339 (2014).

39. Alagapan, S. et al. Modulation of cortical oscillations by low-frequency direct cortical stimulation is state-dependent. PLoS Biol. 14, e1002424 (2016).
40. Schwartz, Z. P. \& David, S. V. Focal suppression of distractor sounds by selective attention in auditory cortex. Cereb. Cortex. 28, 323-339 (2018).

41. Leuchter, A. F., Cook, I. A., Jin, Y. \& Phillips, B. The relationship between brain oscillatory activity and therapeutic effectiveness of transcranial magnetic stimulation in the treatment of major depressive disorder. Front. Hum. Neurosci. 7, 37 (2013). 